\title{
Formación continua de docentes durante la contingencia sanitaria desde la perspectiva de directivos escolares
}

\author{
Teacher professional development during the sanitary contingency from the principals' perspective \\ Karen Patricia Rivera Ceseña \\ Graciela Cordero Arroyo \\ Sergio Reyes-Angona
}

\begin{abstract}
RESUMEN
El campo educativo es uno de los más afectados por la COVID-19. Los docentes se han tenido que adaptar para desarrollar sus clases en ambientes completamente distintos a los habituales. A pesar de la abundante investigación de las nuevas condiciones educativas, un aspecto poco explorado ha sido la oferta de formación recibida por los docentes en este contexto. El objetivo de este artículo es analizar la percepción y valoración de directivos escolares mexicanos de educación primaria respecto a la oferta de formación continua a los docentes durante los meses de contingencia sanitaria, y la manera en que han atendido dicha oferta o impulsado otras acciones. La investigación es exploratoria y tiene un enfoque cualitativo. Se aplicaron entrevistas semiestructuradas a siete directivos de educación primaria de diferentes regiones del país, y su contenido se analizó de forma cualitativa. Como hallazgo principal se constata la multiplicación de la oferta formativa autoadministrable y el impulso paralelo dado por colectivos docentes a las iniciativas de autoformación y formación entre pares. Se concluye la necesidad de combinar dos componentes para la formación docente: 1) la formación autoadministrable, y 2) el acompañamiento a los docentes en sus procesos formativos, con especial atención a su práctica profesional.
\end{abstract}

Palabras clave: educación a distancia, formación autoadministrable, formación docente, mentoría, pandemia.

\section{ABSTRACT}

The educational field is one of the most affected by COVID-19. Teachers have had to adapt to develop their classes in completely different environments from the usual ones. Despite the abundant investigation of the new educational conditions, a little explored aspect has been the offer of training received by teachers in this context. The goal of this article is to analyze the perception and opinion of Mexican primary school administrators regarding the offer of continuous training to teachers during the months of health contingency, and the way in which they have attended to said offer or promoted other actions. The research is exploratory and has a qualitative approach. Semi-structured interviews were applied to seven directors of elementary education from different regions of the country, and their content was analyzed qualitatively. The main finding is the multiplication of the self-administered training offer and the parallel impulse given by teaching groups to self-training and peer training initiatives. It concludes on the necessity to combine two components for teacher training: 1) the self-administered training offer, and 2) the accompaniment of teachers in their training processes, with special attention to their professional practice.

Keywords: online education, self-administered training, teacher training, mentoring, pandemics. 


\section{INTRODUCCIÓN}

El campo educativo es uno de los más afectados por el confinamiento sanitario a causa de la COVID-19. Con el cierre de las escuelas, los docentes han tenido que afrontar, entre otros desafíos, la adaptación abrupta de sus clases a la modalidad en línea o el manejo de herramientas y ambientes digitales, así como la inestabilidad en las condiciones laborales y económicas de las familias que hace evidentes las brechas de acceso a la tecnología, por mencionar algunos (Álvarez, 2020; UNICEF, 2020; Mancera, Serna y Barrios, 2020).

En el año 2020 se publicaron las primeras investigaciones de lo sucedido en las escuelas en el contexto de la pandemia (Reimers y Schleicher, 2020), respecto a diferentes objetos de estudio: enseñanza y aprendizaje en línea (Guerrero, Noroña, Betancourt y Betancourt, 2020); políticas educativas frente a la COVID-19 (Navarrete, Manzanilla y Ocaña, 2020); propuestas, retos, ventajas y desventajas del uso emergente de la modalidad a distancia (Burgos-Videla, Vázquez-Cano, López-Meneses y Adaos-Orrego, 2021); condiciones de las escuelas, evaluación de la estrategia para la continuidad de la educación, recursos utilizados para brindar el servicio educativo, apoyos al profesorado y estrategias para la reapertura de las escuelas (Reimers y Schleicher, 2020).

En México, la Comisión Nacional para la Mejora Continua de la Educación (Mejoredu) publicó los resultados de un estudio a nivel nacional. Con apoyo de cuestionarios en línea se recogieron las experiencias y aprendizajes de 193,749 docentes, directivos, estudiantes y padres y madres de familia de educación básica, durante los primeros

Karen Patricia Rivera Ceseña. Universidad Autónoma de Baja California, México. Es maestrante en Ciencias Educativas del Instituto de Investigación y Desarrollo Educativo (IIDE) y licenciada en Ciencias de la Educación por la UABC. Ha colaborado como asistente de docencia en educación superior, así como en proyectos de investigación nacionales e internacionales en la línea de formación inicial y continua del profesorado. Es diseñadora y facilitadora de programas de formación (cursos, talleres y diplomados) para docentes y estudiantes de educación básica y superior. Correo electrónico: a340734@uabc.edu.mx. ID: https://orcid.org/0000-0002-3993-0024.

Graciela Cordero Arroyo. Profesora-investigadora titular del Instituto de Investigación y Desarrollo Educativo de la Universidad Autónoma de Baja California, México. Es licenciada en Pedagogía por la UNAM, maestra en Educación por la Universidad de Harvard y doctora en Filosofía y Ciencias de la Educación por la Universidad de Barcelona. Es miembro del Sistema Nacional de Investigadores. Fue directora del IIDE-UABC del 2003 al 2011. Editora fundadora de la Revista Electrónica de Investigación Educativa. Recientemente ha sido nombrada presidenta del Observatorio Internacional de la Profesión Docente que gestiona la Universidad de Barcelona. Correo electrónico: gcordero@uabc.edu.mx. ID: https://orcid.org/0000-0001-8567-1732.

Sergio Reyes-Angona. Universidad Autónoma de Baja California, México. Es doctor en Innovación Educativa por el Tecnológico de Monterrey (2019) y doctor en Innovación Didáctica en la Sociedad del Conocimiento por la Universidad Complutense de Madrid (2015). Investigador Nivel I del Sistema Nacional de Investigadores. Actualmente estudia un posdoctorado en el Instituto de Investigación y Desarrollo Educativo (IIDE) de la Universidad Autónoma de Baja California (UABC) y es profesor de cátedra en la maestría en Tecnología Educativa del Tecnológico de Monterrey con el diseño de una materia optativa: Escritura Académica y Tecnología Digital. Correo electrónico: sergioreyes@uabc.edu.mx. ID: https://orcid.org/0000-0003-2329-2352. 
meses de la contingencia. Los temas abordados se organizaron en tres dimensiones: 1) ¿Cómo vivió la comunidad escolar la experiencia de educación durante la contingencia sanitaria?, 2) ¿Qué retos y dificultades enfrentaron los actores escolares durante la contingencia sanitaria? y 3) ¿Qué sugieren los actores para el regreso a clases y para futuras situaciones de emergencia? (Mejoredu, 2020b).

Uno de los primeros trabajos publicados a inicios de la pandemia señala que el profesorado requiere acompañamiento y formación para adecuarse a estas nuevas exigencias (Reimers y Schleicher, 2020). A lo largo del 2020, los gobiernos definieron programas específicos para capacitar a los maestros en el uso de la tecnología con fines didácticos. Es posible observar que, a pesar de la abundante investigación de las nuevas condiciones educativas y del énfasis reiterado en la importancia del maestro en este contexto de emergencia, un aspecto estratégico aún poco explorado ha sido la oferta de formación recibida por los docentes a lo largo del 2020.

Es por ello que esta investigación indaga la percepción de directivos escolares de educación primaria en México respecto a la oferta de formación a docentes en servicio durante los meses de contingencia sanitaria, así como la manera en que los directivos y sus colectivos docentes han atendido dicha oferta o promovido acciones formativas adicionales.

\section{LA FORMACIÓN CONTINUA EN LÍNEA PARA DOCENTES}

La formación continua de los docentes se ha considerado una pieza angular de la mejora educativa, ya que se reconoce que "este proceso constituye la vía por la que el sistema renueva sus prácticas, cuestiona sus tradiciones, acepta nuevas visiones teóricas, se abre al conocimiento y se revitaliza" (Zaldívar, Quiroz y Medina, 2017, p. 91). Del año 2010 a la actualidad, la formación en línea de docentes ha acelerado su expansión ante los beneficios que se le atribuyen (Dulude, Allman, Maile y Royce, 2020). Entre ellos, como argumentan Dede, Ketelhut, Whitehouse, Breit y McCloskey (2009), la modalidad en línea permite la flexibilidad en tiempos y espacios, la promoción de trabajo en redes y la posibilidad de distribuir masivamente los contenidos de la formación.

Con el cierre de las escuelas por la contingencia sanitaria, la formación en línea además de útil se tornó indispensable y se ha enfocado principalmente en los diferentes aspectos tecnológicos y pedagógicos vinculados a cómo enseñar en línea (Dulude et al., 2020). Para poder atender de forma urgente a un número muy amplio de docentes (escalabilidad) y, además, para facilitarles el acceso y gestión en línea de esa formación ante la saturación y complejidad creciente de sus horarios de trabajo en la contingencia (flexibilidad), ha proliferado la formación en línea autoadministrable con una amplia variedad de recursos y actividades, como videotutoriales, webinars y múltiples cursos no-tutorados. 
Reimers y Schleicher (2020) señalan que uno de los aspectos necesarios para favorecer la continuidad educativa durante la contingencia ha sido y seguirá siendo la atención y apoyo a los docentes. En el contexto de la emergencia sanitaria, su propuesta va más allá del uso de recursos autoadministrables y sugieren acciones de acompañamiento como coaching y mentoría, redes de colaboración de pares e instituciones escolares y programas de formación docente en línea sostenida y profunda. Con ello, el objetivo no solo es brindar al profesorado información, sino desarrollar habilidades profesionales para la atención de necesidades emergentes de carácter académico, mental y social de los estudiantes.

De acuerdo con los resultados de investigaciones previas (SREB, 2004; Collins y Liang, 2015; Surrette y Johnson, 2015; Rasmussen y Byrd, 2016; Borup y Evmenova, 2019), para que la formación de profesores en la modalidad en línea sea efectiva debe apoyar la reflexión y mejora de la práctica profesional de los docentes de tal manera que:

- promueva la colaboración entre participantes y la generación de comunidades de aprendizaje;

- incluya actividades que se vinculen con el contexto profesional de los docentes;

- implique un aprendizaje activo adecuado a sus necesidades, con espacios para reflexionar respecto a su actuar en el aula, y

- brinde una retroalimentación efectiva y una valoración de su desempeño en el programa formativo.

\section{Política de formación continua del profesorado durante el 2020}

La Dirección General de Formación Continua, Actualización y Desarrollo Profesional de Maestros de Educación Básica (DGFC) de la Secretaría de Educación Pública (SEP) publicó, a inicios de la contingencia, la Estrategia Nacional de Formación Continua (ENF) 2020, documento en el que la formación continua es definida como

...un proceso permanente de adquisición de conocimientos, desarrollo de aptitudes, capacidades y habilidades que le permite al docente una reflexión sistemática de su práctica educativa, para transformarla y lograr mejores aprendizajes en sus alumnas y alumnos, particularmente en este periodo de contingencia sanitaria, que nos ha mostrado carencia de habilidades para aprender a aprender [SEP, 2020, p. 3].

En la ENF 2020 (SEP, 2020) se indican cuatro modalidades para impartir la oferta formativa: 1) en línea, 2) bimodal, 3) presencial con apoyo de TIC y 4) círculos de estudio virtuales. Específicamente, la modalidad en línea se describe como:

Sistema tecnológico de comunicación bidireccional que sustituye la interacción personal en el aula de profesor y alumno como medio preferente de enseñanza, por la acción sistemática y conjunta de diversos recursos didácticos y el apoyo de una organización y tutoría, que propician el aprendizaje independiente y flexible de los estudiantes. En algunos casos, se puede optar por 
formación autogestiva con acompañamiento por medio de círculos de estudio virtuales en el que un facilitador apoyará al profesorado [SEP, 2020, p. 10].

La DGFC adopta una definición de formación en línea en la que los participantes son agentes activos en sus procesos formativos. Se perfila un sistema en el cual el facilitador es un recurso didáctico fundamental, en tanto que su función no solo es la enseñanza sino el acompañamiento.

Si bien la estrategia propone que la formación en línea del profesorado se apoye en un facilitador y se brinde atención técnica (SEP, 2020), la oferta formativa que figura en el portal oficial de la DGFC no coincide con lo indicado en el documento de la ENF (SEP, 2020), ya que predomina el formato de formación orientada hacia el trabajo individual sin acompañamiento. Al 14 de diciembre del 2020, toda la oferta publicada en el portal se dividía en seis ejes, todos con las características alineadas al formato autoadministrable:

- Talleres emergentes de formación docente. Tres talleres ofertados por Mejoredu.

- Cursos abiertos de formación continua para la contingencia. Programas y recursos ofertados por las autoridades educativas estatales (AEE).

- Proyectar la enseñanza. Se muestran tres enlaces a este curso (según los actores educativos) y se encuentran disponibles dos documentos de materiales de trabajo.

- Cursos abiertos de aprendizaje en línea. Corresponde a la colección Aprendizajes clave y son cursos en línea, masivos y abiertos (MOOC, por sus siglas en inglés).

- Programa@prende 2.0. Sitio web que presenta la "Estrategia digital en educación [...] que busca fomentar el uso de las TIC para fomentar el desarrollo de las habilidades digitales y el pensamiento computacional" (Coordinación General @prende.mx, 2016, párr. 1);

- Centro Virtual de Formación Continua, CEVIF. Se identifican un webinar abierto en YouTube y cursos administrados por la Universidad Abierta y a Distancia de México, y por la UNICEF.

Otra opción de formación fue la Estrategia de Educación a Distancia: transformación e innovación para México, iniciativa implementada a partir de abril del 2020 por la SEP en colaboración con Google for Education y YouTube, que consiste en la transmisión de webinars (Google, s.f.) para capacitar a los maestros en el uso de estos recursos.

Aunque en la ENF (SEP, 2020) y en las descripciones de algunas acciones formativas de la federación se utiliza el término autogestivo, en este artículo se propone el término autoadministrable para referir a la formación no-tutorada ofrecida por las instituciones educativas y organismos gubernamentales, ya que el concepto de autogestión tiene implicaciones diferentes. La formación autogestiva mediada por tecno- 
logía se centra en el aprendiz, que es quien promueve, evalúa y se responsabiliza de su propio aprendizaje, a través de procesos en que se involucra, participa, reflexiona, es propositivo, proactivo, organizado e implementa su capacidad de metacognición (Ponce, 2016). Los principios de trabajo participativo y autoregulado están presentes en la formación no-tutorada (Guarneros-Reyes, Espinoza-Zepeda, Silva y SánchezSordo, 2016), en la formación autónoma con acompañamiento de un facilitador (Ponce, 2016) y en el aprendizaje autodirigido (Perassi, Carrere, Miyara, Pita, Waigandt y Añino, 2017), principios que no se reconocen en la formación autoadministrable asociada con la capacitación o el entrenamiento de los docentes.

\section{Método}

La investigación, exploratoria y con un enfoque cualitativo, contó con la participación de siete directores (tres mujeres y cuatro hombres) de educación básica en cuatro estados de México: tres de Baja California, dos de Sonora, uno de Puebla y una en Nayarit. La muestra, además de abarcar diferentes regiones del país (noroeste, este y centro), incluye escuelas de diversos entornos (dos de entorno rural, dos urbano y cuatro semiurbano) y estructura administrativa-financiera (estatales y federales). Se enfoca en escuelas ubicadas en contextos de vulnerabilidad socioeconómica, pues este perfil de escuelas ha sido el más desafiado por la brecha digital para abordar la transición a una educación a distancia. La totalidad de los participantes reportó contar con posgrado: cinco indicaron que su último grado de estudios es maestría, uno doctorado y uno posdoctorado. La antigüedad en el cargo directivo varió desde uno hasta diez años. En la tabla 1 se muestran las características de cada directivo.

Seis de los siete participantes comentaron que además de ser directores escolares también desempeñan otras actividades profesionales. Los D2 y D5 indicaron ser asesores técnicos de directores; D4 es docente de grupo en primaria; D6 es académico de escuela Normal, y D3 afirmó:

...formo parte de la Red Estatal de Asesorías para Docentes. Nos encargamos de dar actualización, a través de talleres, a los docentes de todo el estado [...] Esta nueva administración de la Secretaría de la Educación no ha tenido una organización al respecto, entonces, no he desempeñado esa parte. También estoy capacitado para ser tutor acompañante de los docentes noveles de educación básica; además de eso, trabajo en la escuela Normal, como asesor de tesis, investigo en un cuerpo académico y también doy clases a grupos [D3-1,2].

Para la recolección de datos se utilizaron entrevistas semiestructuradas, con apoyo de un guion organizado de acuerdo a tópicos clave, pero flexible al devenir del testimonio de cada participante. Los tópicos que guiaron las entrevistas fueron los siguientes:

1) Perfil y contexto, con información general del participante y contexto de la escuela donde se desempeñaba como directivo. 
Tabla 1. Características de los participantes (directivos escolares de México).

\begin{tabular}{cccccc} 
Participante & Sexo & Entidad federativa & $\begin{array}{c}\text { Último grado } \\
\text { de estudios }\end{array}$ & $\begin{array}{c}\text { Años de servicio } \\
\text { en cargo directivo }\end{array}$ & $\begin{array}{c}\text { Contexto de la } \\
\text { escuela que dirige }\end{array}$ \\
\hline D1 & Femenino & Baja California & Maestría & 10 & Rural \\
\hline D2 & Masculino & Sonora & Maestría & 4 & Urbano marginado \\
\hline D3 & Masculino & Baja California & Maestría & Urbano marginado \\
\hline D4 & Masculino & Puebla & Maestría & Urbano marginado \\
\hline D5 & Femenino & Sonora & Posdoctorado & Urbano & Urbano \\
\hline D6 & Masculino & Baja California & Maestría & 4 & Rural
\end{tabular}

Fuente: Construcción personal.

2) Organización del colectivo docente para brindar el servicio educativo durante la contingencia sanitaria.

3) Oferta y características de la formación continua de docentes a lo largo de la contingencia.

La recolección de datos se llevó a cabo durante las primeras semanas de octubre del 2020, es decir, a más de seis meses del inicio del confinamiento preventivo, y en los primeros meses del ciclo escolar 2020-2021 impartido a distancia por medio de diversos recursos. Dado el contexto de distanciamiento social, las entrevistas se llevaron a cabo por videollamada en la plataforma Google Meet, medio que HernánGarcía, Lineros-González y Ruiz-Azarola (2020) recomiendan para la adaptación de la investigación cualitativa a las condiciones de confinamiento.

Las sesiones fueron audio-grabadas (con el consentimiento de los participantes) y transcritas con la finalidad de proceder al análisis de la información. En atención al anonimato de los directivos, para efectos de este reporte se les asignó una clave de participante. Estas claves son utilizadas en la sección de resultados para hacer referencia a los comentarios emitidos por cada director y se identifican de la siguiente manera: D1-5, donde D1 es por Director 1, y 5 (número después del guion) corresponde al número de página en la transcripción donde se ubica el extracto específico de texto.

El proceso analítico de los datos se llevó a cabo a partir de los seis pasos propuestos por Cáceres (2003):

1. Selección del objeto de análisis: se partió del objetivo de la investigación, orientado a analizar la oferta de formación continua del profesorado durante la contingencia sanitaria.

2. Desarrollo del preanálisis: primera revisión, lectura y organización de los datos (siete transcripciones).

3. Definición de las unidades de análisis: a partir de la utilización de matrices en las que se colocaron los fragmentos de contenido que se identificó que se alineaban al objetivo del estudio; se hizo una codificación y categorización a 
partir de las temáticas del guion de la entrevista, así como de carácter inductivo (Cáceres, 2003).

4. Establecimiento de reglas de análisis y códigos de clasificación: se designaron los criterios para codificar las unidades de análisis y se acordaron las etiquetas de los códigos.

5. Desarrollo de categorías: una vez que se tuvo la codificación, los códigos se ordenaron de acuerdo al tipo de información que aportaban, con lo cual se definieron las categorías.

6. Integración final de los hallazgos: representada en los resultados y conclusiones de este artículo.

\section{Resultados}

Como producto del análisis cualitativo del contenido de las siete entrevistas se establecieron 19 códigos, organizados en cuatro categorías, cuyas definiciones se presentan en la tabla 2.

A continuación se presentan los resultados de la investigación organizados de acuerdo a las cuatro categorías de análisis: contexto escolar, oferta formativa, formación ideal e iniciativas de los directivos.

\section{Contexto escolar}

Las características organizacionales de las escuelas dirigidas por los participantes son diversas. Trabajan en escuelas tanto de sostenimiento estatal como federal, de turno matutino, vespertino y de tiempo completo, de contextos urbanos, rurales y urbanos marginados y de tamaños (en términos de matrícula) bastante diferenciados, desde 156 estudiantes en la más pequeña hasta 350 en la más grande (véase tabla 3).

La mayoría de los participantes reportó que en estos centros escolares predomina un contexto de vulnerabilidad y un nivel socioeconómico bajo. Esta situación ha representado uno de los principales retos para la implementación de la educación a distancia, dada la carencia de recursos tecnológicos y económicos para la cobertura del pago de servicios de telecomunicaciones (internet inalámbrico y datos móviles) y adquisición de dispositivos electrónicos.

Como consecuencia de tal situación, los directores señalaron que sus equipos docentes se encontraban limitados respecto a las herramientas digitales para llevar a cabo la educación a distancia. En la tabla 4 se muestra un concentrado de las herramientas y recursos que el profesorado ha utilizado durante el periodo de confinamiento.

Se observa que, de acuerdo a los directores escolares entrevistados, hasta en cinco de las escuelas se implementaron recursos como Google Classroom y Google Meet. No obstante, el uso de estas herramientas estaba limitado a las posibilidades económicas de los padres de los estudiantes. Generalmente, el trabajo tenía que ser 
Tabla 2. Definición de categorías obtenidas en el proceso de análisis de las entrevistas realizadas.

\begin{tabular}{|c|c|c|}
\hline Categoría & Definición & Códigos \\
\hline $\begin{array}{l}\text { Contexto } \\
\text { escolar }\end{array}$ & $\begin{array}{l}\text { Características contextuales de la comunidad y del centro } \\
\text { escolar, asociadas a las técnicas, metodologías y } \\
\text { herramentas implementadas tanto para mantener contacto } \\
\text { con estudiantes y padres de familia como para establecer } \\
\text { los procesos de enseñanza y aprendizaje }\end{array}$ & $\begin{array}{l}\text { 1. Contexto socioeconómico de los estudiantes } \\
\text { 2. Aplicaciones, herramientas y plataformas } \\
\text { utilizadas } \\
\text { 3. Retos } \\
\text { 4. Planta docente }\end{array}$ \\
\hline $\begin{array}{l}\text { Oferta } \\
\text { formativa }\end{array}$ & $\begin{array}{l}\text { Características de oferta formativa dirigida a los } \\
\text { profesores de educación básica durante el } \\
\text { confinamiento preventivo }\end{array}$ & $\begin{array}{l}\text { 5. Formación impartida por las autoridades } \\
\text { educativas } \\
\text { 6. Formación impartida por proveedores externos } \\
\text { 7. Propósito de la formación } \\
\text { 8. Contenido de la formación } \\
\text { 9. Dispositivos formativos } \\
\text { 10. Entrega de Constancias } \\
\text { 11. Obligatoriedad de la oferta } \\
\text { 12. Exceso de oferta }\end{array}$ \\
\hline $\begin{array}{l}\text { Formación } \\
\text { ideal }\end{array}$ & $\begin{array}{l}\text { Cualidades deseadas en un programa de } \\
\text { formación docente en línea, desde la perspectiva } \\
\text { de los directivos escolares }\end{array}$ & $\begin{array}{l}\text { 13. Tipo de dispositivo formativo } \\
\text { 14. Función del facilitador } \\
\text { 15. Tamaño de grupos } \\
\text { 16. Duración de la formación } \\
\text { 17. Diagnóstico de necesidades formativas }\end{array}$ \\
\hline $\begin{array}{l}\text { Iniciativas de } \\
\text { los directivos }\end{array}$ & $\begin{array}{l}\text { Actividades formativas emprendidas individual o } \\
\text { colectivamente por el personal docente y directivo }\end{array}$ & $\begin{array}{l}\text { 18. Autoformación } \\
\text { 19. Formación entre pares/colectivo }\end{array}$ \\
\hline
\end{tabular}

Fuente: Construcción personal.

Tabla 3. Características de los centros escolares reportadas por los directivos entrevistados.

\begin{tabular}{|c|c|c|c|c|c|}
\hline Participante & Sostenimiento & Turnos & Contexto & Tipo de organización & Matrícula \\
\hline D1 & Estatal & Ambos & Rural & Sin dato & 180 y 360 \\
\hline D2 & Sin dato & Tiempo completo & Urbano marginado & Completa & 175 \\
\hline D3 & Federal & Tiempo completo & Urbano marginado & Completa & 156 \\
\hline D4 & Federal & Vespertino & Urbano marginado & Completa & 272 \\
\hline D5 & Estatal & Sin dato & Urbano & Completa & 350 \\
\hline D6 & Federal & Matutino & Urbano & Completa & 205 \\
\hline D7 & Sin dato & Matutino & Rural & Completa & 313 \\
\hline
\end{tabular}

Nota: la participante D1 reportó desempeñar el puesto de directora en un plantel y de encargada de despacho en otro.

Fuente: Construcción personal.

complementado con actividades en material impreso en cuadernillos o consignas por medio de WhatsApp o Facebook, puesto que estas redes sociales suelen estar incluidas en los servicios de datos móviles. En este sentido, los docentes se vieron en la necesidad de diseñar e implementar distintas estrategias que se adaptaran a las condiciones y recursos del alumnado y los padres de familia. 
Tabla 4. Herramientas utilizadas por los docentes para la educación a distancia reportadas por los directivos escolares entrevistados.

\begin{tabular}{lcccccccc} 
& \multicolumn{9}{c}{ Participante } & Total \\
\cline { 2 - 9 } \multicolumn{1}{c}{ Herramienta/recurso } & D1 & D2 & D3 & D4 & D5 & D6 & D7 & N \\
\hline Whats App & $\checkmark$ & $\checkmark$ & $\checkmark$ & $\checkmark$ & $\checkmark$ & $\checkmark$ & $\checkmark$ & 7 \\
\hline Cuadernillos (material impreso) & $\checkmark$ & $\checkmark$ & $\checkmark$ & & $\checkmark$ & $\checkmark$ & $\checkmark$ & 6 \\
\hline Aprende en Casa (I y/o II) & & $\checkmark$ & & $\checkmark$ & $\checkmark$ & $\checkmark$ & $\checkmark$ & 5 \\
\hline G Suite (Classroom, Meet, etc.) & & & $\checkmark$ & $\checkmark$ & $\checkmark$ & & $\checkmark$ & 5 \\
\hline Facebook & $\checkmark$ & $\checkmark$ & $\checkmark$ & & & & & 3 \\
\hline Zoom & $\checkmark$ & $\checkmark$ & & & $\checkmark$ & & & 2 \\
\hline Libro de texto & & & & & & $\checkmark$ & & 1 \\
\hline ClassDojo & & $\checkmark$ & & & & 1 \\
\hline
\end{tabular}

Fuente: Construcción personal.

Los profes en estos momentos tienen que estar preparados, ya visualizados de cuáles son las estrategias que van a utilizar para los que están trabajando con teléfono [...] O sea, tienen que hacer lo mismo en diferentes planeaciones tanto para el que está en teléfono, como para el que está en WhatsApp... hay unos que están en Facebook [D1-3].

Aunque cinco de los directores refirieron el uso de la estrategia nacional de Aprende en casa I y II, ${ }^{1}$ la mayoría indicó que, en acuerdo con el equipo docente, esta se consideró como un recurso de apoyo y no como herramienta eje, por la saturación e inadecuación de sus contenidos para los niveles cognitivos de los estudiantes. En algunos casos incluso se suspendió por completo el uso de dichos programas televisivos. En relación a este aspecto, uno de los participantes señaló:

Hemos optado por basar nuestras planificaciones, no en los programas del Aprende en casa II, porque en este diagnóstico que tenemos, solo el treinta y cinco por ciento de nuestras familias tienen televisión con las condiciones que se requieren. El sesenta y cinco por ciento no la tienen, entonces era como ilógico basar nuestra estrategia de enseñanza en un modelo televisivo que no iban a tener la oportunidad, tampoco a través de Internet, porque ahí las cuestiones se agudizan más, pero sí a través de esta herramienta que ha sido una maravilla y que en la pandemia se ha convertido como en la protagonista, que es la aplicación del WhatsApp [D4-12].

Los resultados indican que el contexto escolar y, específicamente, la situación económica de los padres de familia y de los estudiantes han definido, en gran medida, el tipo de actividades de enseñanza y aprendizaje que los docentes han puesto en marcha para dar continuidad al servicio educativo. En cada caso, el docente ha requerido implementar adecuaciones de distintos medios de acuerdo a las posibilidades particulares de los niños y niñas.

1 Aprende en casa es la estrategia propuesta a nivel federal para dar continuidad al servicio educativo, a través de programación televisiva con los contenidos curriculares de cada grado escolar. 
Asimismo se evidenció que el uso de la estrategia Aprende en Casa (I y II) se consideró poco pertinente para el cumplimiento de los objetivos académicos, por lo que se optó, en la mayoría de los casos, por complementarlo con actividades interactivas (uso de WhatsApp) y adecuadas a las condiciones contextuales y capacidades de los educandos (uso del cuadernillo impreso).

\section{Oferta formativa}

En relación con la oferta formativa se identificaron tipos de proveedores, distintos propósitos, contenido y dispositivos formativos.

Los participantes reconocen dos tipos de instancias involucradas en la producción de la oferta: autoridades educativas y proveedores externos. Dentro de las primeras se encuentran la SEP, las AEE y las supervisiones o inspecciones escolares, y por parte de los proveedores externos se hizo mención del Sindicato Nacional para Trabajadores de la Educación (SNTE), universidades, consultorías, secretarías de gobierno (de Salud y de Seguridad), Fundación Slim (aprende.org), Asociación Civil Únete, entre otras (ver figura 1).

De acuerdo con uno de los directores, en relación con la oferta de proveedores externos, "lo que la SEP [...] ha realizado es, precisamente, ese convenio que tiene con algunas de las empresas" (D2-16), ya que si bien varios de los programas formativos eran diseñados y administrados por empresas, fundaciones, asociaciones civiles o instituciones de educación superior (IES), estos se ofertaban en colaboración con las autoridades educativas.

En cuanto a los propósitos formativos, se identificaron cuatro orientaciones: 1) capacitar, en relación con el manejo de herramientas, protocolos de acción y desarrollo de habilidades; 2) informar, respecto a decisiones institucionales, estrategias

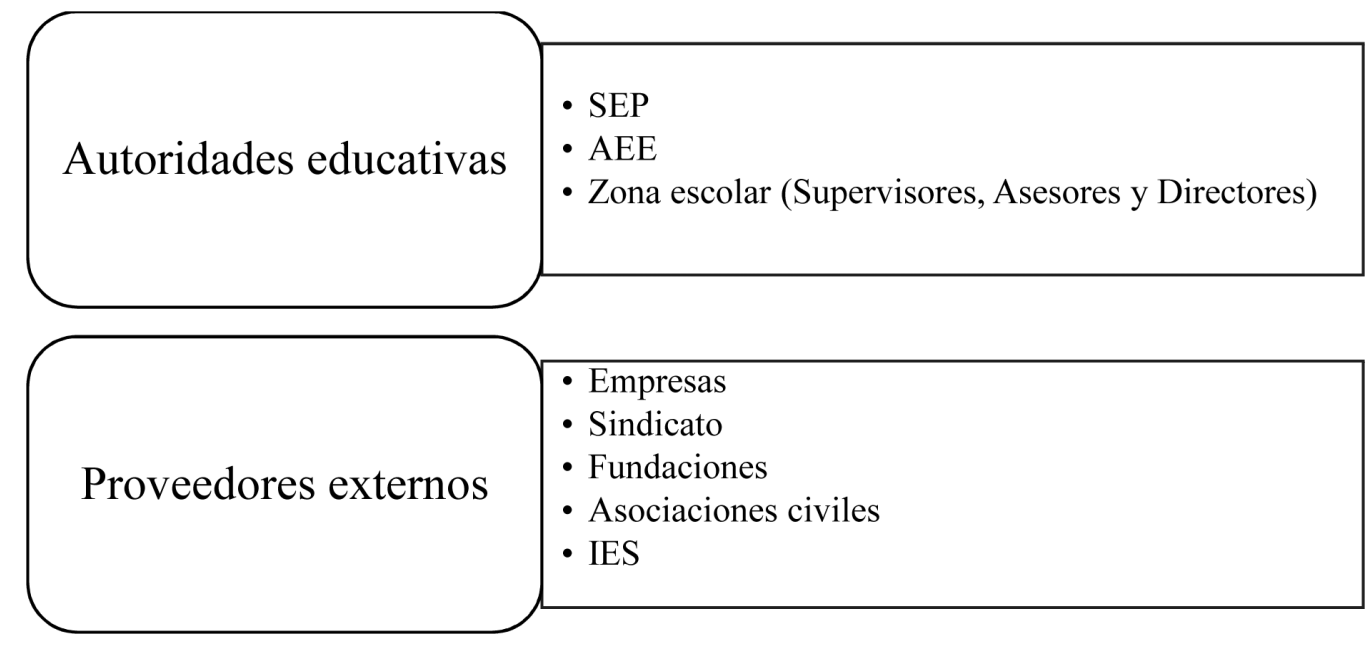

Figura 1. Instancias formadoras identificadas por los participantes.

Fuente: Construcción propia. 
a seguir o herramientas propuestas para la enseñanza y seguimiento; 3) acompañar, para brindar atención al docente por parte del director, asesor técnico o supervisor escolar, y 4) reflexionar con pares, a partir de compartir, revisar y analizar las situaciones experimentadas por los maestros.

Dichos propósitos son abordados por distintos agentes formativos. Mientras que capacitar e informar corresponden, principalmente, a las autoridades educativas federales y estatales, las acciones formativas orientadas al acompañamiento y reflexión del profesorado generalmente se ven reflejadas en iniciativas de la zona escolar (supervisores, asesores y directores) y del mismo colectivo docente (ver figura 2).

\begin{tabular}{|c|c|c|}
\hline $\begin{array}{c}\text { Colectivo docente } \\
\text { Zona Escolar }\end{array}$ & $\begin{array}{c}\text { Autoridad educativa estatal } \\
\text { y federal }\end{array}$ \\
\hline Reflexionar & & \\
\hline \multicolumn{3}{|c|}{ Acompañar } \\
\hline \multicolumn{3}{|c|}{ Capacitar } \\
\hline
\end{tabular}

Figura 2. Propósitos de la formación, según instancias o agentes formativos.

Fuente: Construcción propia.

El contenido de la formación que predominó y que fue referido por todos los participantes corresponde a las temáticas vinculadas al uso de herramientas y desarrollo de habilidades digitales, como el uso de las aplicaciones de G Suite (Google for Education), Microsoft Teams, configuración de aulas virtuales, recursos de Facebook, entre otras. En la tabla 5 se concentran los contenidos formativos mencionados por los participantes.

Se reconocen distintos dispositivos de formación: cursos, talleres, diplomados, webinars y trabajo entre pares, esta última como iniciativa de los directivos y sus colectivos docentes, tal como se explica con más detalle en la última categoría del estudio. Todos los directores mencionaron que se les ha invitado a atender oferta formativa autoadministrable, principalmente los seminarios virtuales relacionados con las herramientas de Google for Education y algunos MOOC. Desde su perspectiva, estos dispositivos suelen presentar tanto ventajas como desventajas:

De repente se justifica esto de la autogestión para que el docente no se sienta estresado en que hay fechas límite para la entrega de las tareas, porque en ocasiones eso es lo que impide que el docente tenga esa oportunidad de la formación continua. [...] en este de G Suite, que no tiene un facilitador [...] sino que es de autogestión y va mediando con lo asincrónico de observar los videos cuando tú tengas tiempo, etcétera, etcétera, como que facilita más. Sienten los docentes que es más flexible un modelo así, en donde, incluso, ni siquiera tengan que hacer ningún tipo de tarea para entregar, sino solo como evidencia de aprendizaje, lo que ha permitido que se convierta en una opción mucho más viable para ellos [D4-17]. 
Tabla 5. Temas de formación.

\begin{tabular}{|c|c|c|c|c|c|c|c|c|c|}
\hline Tema & D1 & $\mathrm{D} 2$ & D3 & D4 & D5 & D6 & D7 & $\mathrm{N}$ & $\%$ \\
\hline \multicolumn{10}{|c|}{ Uso de herramientas y desarrollo de } \\
\hline habilidades digitales & $\checkmark$ & $\checkmark$ & $\checkmark$ & $\checkmark$ & $\checkmark$ & $\checkmark$ & $\checkmark$ & 7 & 100 \\
\hline Pedagogía/Didáctica & & $\checkmark$ & & $\checkmark$ & $\checkmark$ & & & 3 & 42 \\
\hline Educación socioemocional & & $\checkmark$ & & & $\checkmark$ & & & 2 & 28 \\
\hline Ciberseguridad & & $\checkmark$ & & & & & $\checkmark$ & 2 & 28 \\
\hline Protocolo de salud & & $\checkmark$ & & & & & & 1 & 14 \\
\hline Otros & & $\checkmark$ & & $\checkmark$ & $\checkmark$ & & & 3 & 42 \\
\hline
\end{tabular}

Fuente: Construcción personal.

Digamos que tuvimos el engagement, tres o cuatro sesiones, pero ya después de eso ya no les parecía atractivo porque se aburrían o se perdían, entonces, no fue tan efectiva esta forma [webinars] [D3-5].

En este contexto, donde se hizo notar la multiplicidad de instancias involucradas, contenidos emergentes y dispositivos de formación, la oferta fue considerada en algunos casos excesiva y abrumadora:

Sentimos también de repente como que un boom de capacitaciones de todo tipo y de todas las instituciones habidas y por haber. Hasta cierto punto [los docentes] se sienten un poquito atiborrados o muy congestionados de capacitación. En ocasiones estamos agendados casi toda la semana con alguna capacitación [D5-5].

Uno de los aspectos que sobresalió fue el compromiso del profesorado. Aun cuando ninguno de los participantes indicó que alguna de las acciones formativas tuviese carácter obligatorio, todos señalaron que tanto ellos, en su rol directivo, como el personal docente en sus escuelas participaron en diferentes acciones, todas de interés para los docentes en el contexto de la pandemia. Uno de los informantes enfatizó:

A veces son invitaciones, pero tú lees y dice "habilidades socioemocionales para los niños en tiempo de la pandemia” ¿Cómo no lo vas a ver, si es lo que estamos viviendo? Ya te enfocas en eso y luego, “¿Cómo utilizar herramientas tecnológicas para hacer divertida tu clase?”, pues ahí vamos y las vemos, entonces, eso te termina agobiando [D2-4].

La oferta formativa federal y estatal se concentró primordialmente en informar y capacitar por medio del formato autoadministrable, en aras de atender las necesidades emergentes de manera masiva para llegar al mayor número posible de docentes. Sin embargo, aunque en algunos casos los directivos han considerado pertinentes los contenidos, también se ha puesto de relieve la saturación de oferta a la que se enfrentaron en los últimos meses.

\section{Formación ideal}

En las sesiones de entrevistas también se recabó información respecto a las cualidades que los directores consideraban deseables en los programas de formación docente, 
específicamente en modalidad en línea. Destacó la tendencia a solicitar formación que contara con acompañamiento de un facilitador e interacción con compañeros; sin embargo, los participantes también consideran que, dadas sus condiciones laborales y personales, la formación autoadministrable es idónea. Por lo tanto, proponen acciones que combinen el trabajo autónomo y flexible con el apoyo de una figura que funja como guía en el proceso formativo:

Tendría que ser autogestionable, porque con tantas actividades que tenemos es difícil estar sobre tiempo; pero tendría que tener un acompañante, porque no todos somos tan disciplinados para continuar con las temáticas que se van proponiendo. Creo que un curso dirigido podría funcionar muy bien para dar este seguimiento, no para fiscalizar o evaluar, sino para ayudarte, porque, sé que muchos se quedan a medias pensando "no cuenta" o "no se van a dar cuenta" o "no entendí esta parte, entonces me bloqueo y no le sigo", entonces, algo que tenga ochenta por ciento de autogestión y el restante de acompañamiento [D3-6,7].

Del mismo modo, los directores refirieron elementos asociados al tamaño de los grupos de participantes en las acciones formativas: "lo ideal sería que fueran cursos estatales en donde fuera una cantidad no tan grande de participantes para poder tener esta interacción" (D1-7). Aunado a ello, comentaron la importancia de contar con formación contextualizada, que considere sus necesidades y condiciones laborales, así como adecuar la duración de las acciones formativas para lograr abarcar los contenidos en profundidad.

En resumen, los participantes reportan que un programa formativo en línea ideal es aquel que:

- Parte de sus necesidades profesionales y contextuales.

- Ofrece flexibilidad y autonomía para la revisión de contenidos.

- Tenga una figura de apoyo para resolver dudas y recibir acompañamiento.

- Favorezca la interacción con pares y el aprendizaje activo.

\section{Iniciativas de los directivos}

A partir de las necesidades emergentes por la contingencia sanitaria, los directivos emprendieron con sus equipos docentes diferentes actividades adicionales con fines formativos, al menos en dos sentidos: 1) autoformación y 2) formación entre pares, en ocasiones concatenados para fortalecer las habilidades y conocimientos adquiridos:

Me autocapacité con Meet viendo tutoriales, viendo videos, preguntándole a la inspectora “¿cómo le hago para presentar?, porque no puedo", mandar la liga, etcétera, etcétera, pero ahí estamos, ya lo aprendimos, ya lo manejamos y lo que sigue es Classroom [D1-5].

Los maestros han estado capacitándose de manera autónoma también en el sentido de estar buscando medios, herramientas y estar ellos de manera autónoma [D5-10].

...[los profesores] miraban algún video de You'Tube en donde daban explicaciones sobre herramientas y nos lo compartían en el grupo de docentes [D1-5]. 
En cuanto a formación entre pares se señalaron iniciativas de directivos y docentes para identificar, compartir y reflexionar acerca de prácticas exitosas y áreas de oportunidad en el contexto de educación a distancia, a partir de las experiencias vividas por cada uno de los profesores.

Respecto a estas acciones, los directivos comentaron ejemplos como: "Nos reunimos todos los viernes los maestros, aprovechamos esos viernes para ver cómo nos fue, hacemos como una reflexión y compartimos experiencias" (D7-7); "hemos dado también seguimiento de manera interna, por así decirlo, a experiencias exitosas que hemos tenido" (D5-5); "lo que sucede en la escuela si yo no lo puedo resolver o algo, tenemos un grupo de WhatsApp de directores, o si no, tenemos un asesor acompañante" (D1-7). Cabe destacar que estas acciones no eran referidas explícitamente por los participantes como actividades formativas, sino como prácticas de apoyo entre ellos.

En el mismo eje, algunos directores comentaron el procedimiento seguido para trabajar con sus docentes:

Trabajé con los docentes para habilitarlos en el uso de la plataforma Classroom, todo mi Consejo Técnico Escolar lo realicé por ese medio con dos intenciones: uno, para facilitarme el proceso de seguimiento con ellos, y dos, para obligar, ayudar o invitar a que utilizaran el Classroom. Esto funcionó bastante porque una vez que nos incorporamos a las clases en línea los profes perdieron el miedo para trabajar con la plataforma [D3-4].

Algunas de estas oportunidades para que los docentes sigan enriqueciendo su formación continua han sido diseñadas por mí. He trabajado con la formación en ellos de lo tecnológico, de los aspectos relacionados con la intervención pedagógica, a través de metodologías como el aprendizaje basado en proyectos, y también la evaluación de carácter formativo [D4-12,13].

Con las maestras de primer grado trabajo dos días a la semana, por las tardes para capacitarlas en las metodologías de métodos globales, de la incorporación del neuroaprendizaje, de la neurociencia, y también ahora de los contextos virtuales [D4-14].

Estas acciones emprendidas como iniciativas de los directivos, además de contribuir a la capacitación y reflexión en temas específicos y emergentes, les han servido para la configuración de redes de apoyo profesional:

De alguna manera, los docentes, como ya se comentó, no se sienten solos, que creo que esa es una de las cosas que tenemos que fortalecer mucho en estos momentos, se sienten acompañados en el proceso no solamente por mí, en esta parte, sino también por todos los docentes, por todo el equipo [D7-13].

Los directivos han explorado distintas estrategias para resolver sus necesidades formativas a través del diálogo, la reflexión, el intercambio de experiencias y la colaboración entre pares. La diversidad del personal docente, respecto a antigüedad en el servicio, actitud ante la contingencia y habilidades digitales permitieron la complementariedad de los perfiles profesionales para la conformación de estas redes de apoyo. 


\section{ConClusiones}

Durante la contingencia sanitaria, la inmersión en la tecnología ha sido una migración obligatoria y forzosa tanto para maestros como para estudiantes. Esta situación hizo evidente las diferencias y brechas sociales y económicas en la población estudiantil. No obstante, el personal docente y directivo ha continuado brindando el servicio educativo con herramientas que hubo que adecuar a las posibilidades económicas y tecnológicas de estudiantes y familias. Los docentes se han adaptado a los recursos con los que cuenta su alumnado y, debido a la diversidad de posibilidades que presenta cada grupo escolar, el profesorado ha explorado distintas estrategias y medios para abordar los contenidos.

La desigualdad social y la brecha digital no se presentan únicamente en México, sino que han sido identificadas también en el contexto internacional, específicamente en los países de América Latina y el Caribe, pues no todos los estudiantes tienen la posibilidad de acceder al servicio de internet, a una computadora o a un teléfono móvil (CEPAL, 2020). Estas situaciones han implicado distintos retos para el profesorado y para los sistemas educativos, presentándose condiciones que están fuera de su control o de su ámbito de impacto directo.

Las autoridades educativas mexicanas federales y estatales han implementado acciones de apoyo a los docentes durante la contingencia, específicamente orientadas a la formación. La SEP y las AEE ofrecieron al profesorado a lo largo del 2020 una formación en línea principalmente autoadministrable. La emergencia por responder a las necesidades básicas e inmediatas del profesorado en el uso de medios digitales para dar continuidad al servicio educativo requirió la puesta en marcha de estrategias masivas que facilitan la autoadministración en línea del contenido, actividades y materiales de los cursos. A pesar del reconocimiento explícito del valor de la figura del facilitador en la formación en línea y de los propósitos de seguimiento y retroalimentación enunciados en la ENF (SEP, 2020), en los hechos esto no se materializó.

Los directivos confirman esta situación y explican que la oferta formativa en estos meses de pandemia se ha presentado en formato autoadministrable. Una de sus ventajas es permitir al profesorado elegir aquella oferta que le es más conveniente y en los horarios en que puede atenderla (Dede et al., 2009). Al mismo tiempo, los maestros expresan confusión acerca de la mejor manera de administrarla, dada la proliferación de oferta definida por la autoridad, pero también la que emerge de sus propios intereses. Ambas terminan por saturar su tiempo de dedicación. Si bien la formación en línea es flexible en términos de horarios, no debe desestimarse que las actividades de formación en línea también implican tiempo, recursos y esfuerzos del profesorado (Dede et al., 2009). 
Se reconoce que en los documentos oficiales y en el vocabulario de los directivos se utiliza el concepto autogestivo. En ambos contextos este concepto se utiliza como sinónimo de formación no-tutorada. En la literatura, la autogestión es un concepto complejo ya que está asociada con la autodirección de la formación y el aprendizaje (Perassi et al., 2017). En la formación autogestiva el aprendiz desarrolla un perfil específico de competencias, con habilidades cognitivas, organizacionales, motivacionales y actitudinales que facilitan su aprendizaje (Ponce, 2016). De ahí que en esta investigación se propone el término autoadministrable para hacer referencia a la capacitación o entrenamiento masivo en el uso de los recursos tecnológicos que no atienden o sostienen el proceso de aprendizaje de los maestros en el contexto de su práctica docente.

En cuanto a las características deseables de los programas formativos, los participantes valoran positivamente la implementación de oferta autoadministrable, pero subrayan también la necesidad de complementarla con el apoyo de una figura facilitadora. Si bien el profesorado aprecia las ventajas de la oferta brindada por las instancias gubernamentales, considera que carece de un elemento fundamental: el acompañamiento.

A modo de resumen, por tanto, se concluye que una oferta de formación en línea adecuada en el contexto de la presente contingencia requiere, al menos, dos componentes: 1) la flexibilidad, disponibilidad y escalabilidad de acciones formativas autoadministrables (de carácter masivo, consultable en horarios y espacios diversos), y 2) el acompañamiento y orientación a los docentes en los procesos de transferencia de esas habilidades a su práctica profesional. Esa estrategia se ve fortalecida por una de las recomendaciones de la Mejoredu (2020a) para favorecer la continuidad educativa durante la contingencia, que se refiere a "Promover oportunidades de formación y desarrollo profesional docente en colectivo" (p. 9).

El pilar fundamental de esta propuesta se vincula con la disposición e iniciativa percibidas en las prácticas reportadas por los directores escolares. Un ejemplo emblemático de esas iniciativas es una de las acciones emprendidas por el D3, quien, a partir de los contenidos de los webinars, atendía dudas particulares que surgían en su colectivo. Del mismo modo, uno de los participantes reconoció explícitamente que "dentro de las responsabilidades que los directores tienen es, precisamente, fortalecer las áreas pedagógicas, en esta dimensión pedagógica de la gestión directiva, para fortalecer los procesos de capacitación de los docentes" (D4-12).

Cabe destacar que, si bien se reconoce la disposición de las figuras directivas para apoyar los procesos formativos de los integrantes de sus colectivos docentes, en una estrategia de formación de este tipo (conjugación de oferta autoadministrable con acompañamiento de directores escolares) se hace necesaria la formación de los directivos tanto en los contenidos como en habilidades para el acompañamiento al profesorado, ya que fungiría como un proceso de mentoría (Gall y Acheson, 2011). 
Estos nuevos escenarios pueden representar un parteaguas en la transición a entornos formativos más flexibles, informales, autónomos y reflexivos, que dejen de lado la figura del docente como un mero receptor, para ser considerado un participante activo en su formación y en la de sus pares, con los que forma redes de apoyo y colaboración para la mejora de sus saberes profesionales.

\section{Agradecimientos}

Este estudio se realizó gracias al apoyo de la convocatoria de Ciencia Básica de CoNACyT México (Proyecto no. A1-S-29890).

\section{REFERENCIAS}

Álvarez, G. (2020, abr. 16). Covid-19. Cambiar de paradigma educativo. Recuperado de: http://www.comie.org.mx/ v5/sitio/2020/04/16/covid-19-cambiar-de-paradigmaeducativo/.

Borup, J., y Evmenova, A. (2019). The effectiveness of professional development in overcoming obstacles to effective online instruction in a college of education. Online Learning Journal, 23(2). Recuperado de: https:/ /olj. onlinelearningconsortium.org/index.php/olj/article/ view/1468.

Burgos-Videla, C., Vázquez-Cano, E., López-Meneses, E., y Adaos-Orrego, R. (2021). Proyecto Difproret: análisis de las dificultades, propuestas y retos educativos ante el COVID-19. International Journal of Educational Research and Innovation, (15), 17-34. doi: 10.46661/ijeri.5145.

Cáceres, P. (2003). Análisis cualitativo de contenido: una alternativa metodológica alcanzable. Psicoperspectivas, 2(1), 53-82. Recuperado de: https://psicoperspectivas.cl/ index.php/psicoperspectivas/article/viewFile/3/1003.

CEPAL [Comisión Económica para América Latina y el Caribe] (2020, ago.). La educación en tiempos de la pandemia de COVID-19. Recuperado de: https://repositorio.cepal.org/bitstream/handle/11362/45904/1/ S2000510_es.pdf.

Collins, L. J., y Liang, X. (2015). Examining high quality online teacher professional development: Teachers' voices. International Journal of Teacher Leadership, 6(1), 18-34. Recuperado de: https://eric.ed.gov/?id=EJ1137401.

Coordinación General @prende.mx (2016,nov.7).Presentación del nuevo Programa@prende 2.0. Recuperado de: https://www.gob.mx/aprendemx/prensa/presentacion-del-nuevo-programa-prende-2-0-ciudad-demexico-a-7-de-noviembre-de-2016?idiom=es.
Dede, C., Ketelhut, D. J., Whitehouse, P., Breit, L., y McCloskey, E. (2009). A research agenda for online teacher professional development. Journal of Teacher Education, 60(8), 8-19. doi: 10.1177/0022487108327554.

Dulude, C., Allman, B., Maile, R., y Royce, K. (2020). Examining a decade of research in online teacher professional development. Frontiers in Education, (5). doi: 10.3389/ feduc.2020.573129.

Gall, M. D., y Acheson, K. (2011). Clinical supervision and teacher development. Preservice and inservice applications (6a. ed.). Estados Unidos: Wiley.

Google (s.f.). Estrategia de educación a distancia: transformación e innovación para México. Recuperado de: https://events. withgoogle.com/educacion-a-distancia/\#content.

Guarneros-Reyes, E., Espinoza-Zepeda, A. J., Silva, A., y Sánchez-Sordo, J. M. (2016). Diseño de un curso autogestivo modular en línea de metodología de la investigación para universitarios. Hamut'ay, 3(2). doi: 10.21503/ hamu.v3i2.1305.

Guerrero, H. I., Noroña, L. E., Betancourt, A., y Betancourt, E. R. (2020). El moderador en línea: una propuesta pedagógica para la mediación en los procesos de enseñanza y aprendizaje en tiempos de COVID 19. Educare, 24(2), 68-96. doi: 10.46498/reduipb.v24i2.1321.

Hernán-García, M., Lineros-González, C., y Ruiz-Azarola, A. (2020). Cómo adaptar una investigación cualitativa a contextos de confinamiento. Gaceta Sanitaria. doi: 10.1016/j.gaceta.2020.06.007 (consulta: 15 jul. 2020).

Mancera, C., Serna, L., y Barrios, M. (2020, abr. 29). Pandemia: maestros, tecnología y desigualdad. Nexos. Recuperado de: https://educacion.nexos.com.mx/?p=2286. Mejoredu [Comisión Nacional para la Mejora de la Educación] (2020a, abr.). 10 sugerencias para la educación 
durante la emergencia por COVID-19. Recuperado de: https://www.gob.mx/cms/uploads/attachment/ file/546270/mejoredu_covid-19.pdf.

Mejoredu (2020b, nov.). Experiencias de las comunidades educativas durante la contingencia sanitaria por covid-19. Educación básica. Recuperado de: https://editorial.mejoredu.gob. $\mathrm{mx} /$ Cuaderno-Educacion-a-distancia.pdf.

Navarrete, Z., Manzanilla, H. M., y Ocaña, L. (2020). Políticas implementadas por el gobierno mexicano frente al Covid-19. Revista Lationamericana de Estudios Educativos (México), (50, esp.), 143-172. Recuperado de: https:// www.redalyc.org/jatsRepo/270/27063237025/movil/ index.html.

Perassi, M., Carrere, C., Miyara, A., Pita, G., Waigandt, D., y Añino, M. (2017). La formación docente autogestionada a través de la investigación-acción. Revista Electrónica de Didáctica en Educación Superior, (13). Recuperado de: http://ojs.cbc.uba.ar/index.php/redes/article/ view/54.

Ponce, M. E. (2016). La autogestión para el aprendizaje en estudiantes de ambientes mediados por tecnología. Diálogos sobre Educación, 7(12), 1-23. doi: 10.32870/dse. v0i12.258.

Rasmussen, C. L., y Byrd, D. R. (2016). Evaluating continued use of an online teacher professional development program with a sustained implementation scale. Journal of Online Learning Research, 2(2). Recuperado de: https:/ / eric.ed.gov/?id=EJ1148611.

Reimers, F., y Schleicher, A. (2020). Schooling disrupted, schooling rethought. How the Covid-19 pandemic is changing education.
Preliminary version. Recuperado de: https://globaled. gse.harvard.edu/files/geii/files/education_continuity_v3.pdf.

SEP [Secretaría de Educación Pública] (2020). Estrategia Nacional de Formación Continua 2020. México: SEP. Recuperado de: http://dgfc.basica.sep.gob.mx/multimedia/ RSC/BASICA/Documento/202005/202005-RSC5F0G5hyVnk-ENFC2020_12052020.pdf.

SREB [Southern Regional Education Board] (2004). Standards for online professional development guidelines for planning and evaluating online professional development courses and programs. Estados Unidos: SREB. Recuperado de: https:// www.sreb.org/search/site/Standards $\% 20$ for $\% 20$ Online\%20Professional\%20Development.

Surrette, T., y Johnson, C. (2015). Assessing the ability of an online environment to facilitate the critical features of teacher professional development. School Science and Mathematics, 115(6), 260-270. doi: 10.1111/ssm.12132.

UNICEF [Fondo de las Naciones Unidas para la Infancia]. (2020, jun. 5). La falta de igualdad en el acceso a la educación a distancia en el contexto de la COVID-19 podría agravar la crisis mundial del aprendizaje. Recuperado de: https:// www.unicef.org/es/comunicados-prensa/falta-igualdad-acceso-educacion-distancia-podria-agravar-crisisaprendizaje.

Zaldívar, J. D., Quiroz, S. A., y Medina, G. (2017). La modelación matemática en los procesos de formación inicial y continua de docentes. IE Revista de Investigación Educativa de la REDIECH, 8(15), 87-110. doi: 10.33010/ ie_rie_rediech.v8i15.63.

Cómo citar este artículo:

Rivera, K. P., Cordero, G., y Reyes-Angona, S. (2021). Formación continua de docentes durante la contingencia sanitaria desde la perspectiva de directivos escolares. IE Revista de Investigación Educativa de la REDIECH, 12, e1188. doi: 10.33010/ie_rie_rediech.v12i0.1188. 